\title{
WORKING MEMORY IN PATIENTS WITH SCHIZOPHRENIA AND BIPOLAR AFFECTIVE DISORDER: QUANTITATIVE OR QUALITATIVE DIFFERENCES?
}

\author{
Danijela Žakić Milas ${ }^{1}$ \& Goran Milas ${ }^{2}$ \\ ${ }^{I}$ University Psychiatric Hospital Vrapče, Divison of Biological Psychiatry and Psycho-Geriatrics, Zagreb, Croatia \\ ${ }^{2}$ Institute of Social Sciences "Ivo Pilar", Centre for the Individual differences research, Zagreb, Croatia
}

received: 3.7.2018;

revised: 17.11.2018;

accepted: 4.12 .2018

\begin{abstract}
SUMMARY
Background: Some authors suggest that working memory may underlie most of cognitive deficits found in schizophrenia and contribute to the most salient features of the disorder. Many authors further believe that, despite the differences in magnitude, profile of cognitive impairment is quite similar across schizophrenia and affective psychosis. To test the hypothesis of profile similarity between SCZ and BPD compared to healthy individuals, we carried out a comparative study applying several working memory tasks.

Subjects and methods: A total of 64 subjects participated in the study, 20 diagnosed with schizophrenia, 18 with bipolar affective disorder and 26 healthy controls. Groups were matched according to age, sex and education, and two clinical groups were also matched according to the number of hospitalizations. To measure working memory we applied se Wisconsin Card Sorting Test (WCST), STROOP task, Trail making test (TMT), Digit span forward and backward tasks. To test the size and profile similarities of the groups, we used ANOVA and Kruskal-Wallis tests on individual measures and on factor scores.

Results: Most indicators of the WCST did not differentiate between the groups, but all of the remaining indicators indicated weaker working memory of the two clinical groups compared to the healthy controls. All applied measures could be reduced to two latent constructs provisionally named WM Attention and WM Capacity. Both clinical groups scored lower on the capacity component than controls, whereas the three groups could not be distinguished according to the attention component. Results provided no evidence of difference in either size or profile of working memory impairment in patients with SCZ and BDP.

Conclusions: The current study determined impairment of WM in patients diagnosed with SCZ and BPD compared to healthy controls. However, no difference was found regarding either the size or the profile of impairment between SCZ and BPD patients.
\end{abstract}

Key words: working memory - schizophrenia - bipolar affective disorder - comparative study

$$
* * * * *
$$

\section{INTRODUCTION}

The research conducted over the last few decades has provided ample evidence of cognitive impairments in psychotic disorders especially in patients diagnosed with schizophrenia (Barch \& Sheffield 2014, Gold et al. 2017), but also in those diagnosed with mood disorders (MacQueen \& Memedovich 2017). Some authors even argue that cognitive deficits are the main and central feature of schizophrenia (SCZ) and other psychosis (Green et al. 2000, Barch 2003). In addition, there is now impressive body of research documenting that cognitive deficits in persons suffering from psychosis may to a large extent be attributable to impairment of working memory (WM). It has been further suggested that WM may underlie most of cognitive deficits found in SCZ and is likely to contribute to the most salient features of the disorder (Goldman-Rakic 1994, Park et al. 1995), even before psychosis actually emerges (Pflueger et al. 2018).

The concept of WM refers to a hypothetical limited capacity system that enables temporary storage and manipulation of information necessary for performing a wide range of cognitive tasks (Baddeley 1986, 2018). $\mathrm{WM}$ is not conceived as a unitary construct but rather a complex system that can be split into an executive com- ponent and at least two temporary storage subsystems. One of them is concerned with speech and sound while the other is visuo-spatial (Baddeley \& Hitch 1974, Baddeley 2018). These three components are conceived as modules comprising processes and storage systems that are tightly interlinked within the module and more loosely across modules. WM is also connected to other cognitive systems such as perception and long-term memory, enabling guidance of the behavior in response to life experiences and environmental demands. WM is therefore crucial for a well-adapted daily life functioning.

While impairments of WM in SCZ have been extensively researched (Forbes et al. 2009, Lee \& Park 2005, Barch 2003), WM in bipolar affective disorder (BPD) received much less attention (Glahn et al. 2006). Studies have indicated that individuals with SCZ are severely impaired on tasks that require a large amount of information to be maintained in WM, even for a short period of time (Barch et al. 2002). However, several studies have demonstrated that their performance on tasks that require retaining only a few items over a short period, with little or no interference is much less impaired (Cohen et al. 1999, Park \& Holzman 1992). Some authors hypothesize that these findings suggest a greater deterioration of central executive in individuals with schizophrenia (Barch 2003). While they can per- 
form rather well on tasks depending on short-term storage buffers alone, performance on tasks demanding involvement of central executive is usually poor. Even tasks requiring modest manipulation of information, or those requiring elimination even of a limited amount of distracting information tend to seriously impair performance in such individuals. Thus, the conditions under which WM of individuals with SCZ is particularly impaired suggest that $\mathrm{SCZ}$ is related to central executive deficit rather than a disturbance in the buffer systems.

Existing studies further suggest that the impairment of WM among persons with SCZ compared to those suffering from BPD is considerably greater (Krabbendam et al. 2005, Hill et al. 2013). However, despite the differences in magnitude, many authors believe that profile of cognitive impairment is quite similar across schizophrenia and affective psychosis (Tamminga et al. 2014, Barch \& Sheffield 2014).

These findings are somewhat inconsistent with the notion of SCZ and BPD being two clearly distinct entities. Some authors question this common assumption based on the evidence suggesting that these syndromes may share common susceptibility genes (Craddock et al. 2006, Maier et al. 1993).

We can thus conclude that there is an ongoing dispute among researchers whether the differences in WM between persons suffering from SCZ and those with BPD are just of quantitative or also of qualitative nature (Barch \& Sheffield 2014). Recently, cognitive impairment found in persons with SCZ has been associated with the lack of cognitive control and the inability to store the information about the goal that would guide behavior in WM (Barch \& Sheffield 2014, Braver et al. 2009, Edwards et al. 2010). Focusing attention is crucial to actively maintain goal relevant information in WM. According to Engle (2002), WM capacity does not necessarily imply a larger memory store but almost without exception, a greater ability to control attention and to avoid distraction. Authors attribute cognitive deficits regarding goal information representation to the malfunction of the dorsolateral prefrontal cortex and its communication with other brain regions as well as to disturbance in the function of neurotransmitter systems (Lesh et al. 2011).

Some authors believe that the same mechanisms causing malfunction in WM among persons with SCZ are responsible for WM deficits in those with $\mathrm{BPD}$, and that the differences between the two groups are just quantitative (Barch \& Sheffield 2014). However, longitudinal trajectory of WM deterioration in SCZ is different than the one found in BPD patients (Lewandovski et al. 2011) which casts doubt on such a hypothesis.

If, as hypothesized, SCZ and BPD share a common mechanism of WM impairment, then the profile of WM deficits should be mostly similar in these two groups. However, if persons diagnosed with SCZ and those diagnosed with BPD show marked difference in profile of WM deficits compared to healthy people, it could speak in favor of existence of different underlying mechanisms.

\section{Study Aim}

The main aim of the current study was to compare the profile of WM impairment of persons diagnosed with SCZ and those with BPD. To test if the WM impairment is similar in those with SCZ and BPD compared to healthy individuals, we included a number of heterogeneous indicators of WM. In this way we could also identify areas of WM in which persons diagnosed with SCZ show different deficits compared to those with a diagnosis of BPD.

\section{SUBJECTS AND METHODS}

\section{Subjects}

A total of 64 participants took part in the study. Of them 26 were diagnosed with Schizophrenia (SCZ), 18 with Bipolar affective disorder (BPD) and the rest 20 were healthy controls. Patients with SCZ and BPD were admitted to the University Hospital Vrapče in Zagreb and were diagnosed according to the ICD-10 (WHO, 1993). Only patients at the initial stage of the disease were included in the study. The study included male and female subjects aged between 24 and 49 years with at least finished elementary school. The study was approved by the Medical Ethics Committee of the University Hospital Vrapče. All participants signed an informed consent after they were provided with appropriate information.

\section{Group equivalence}

The three groups were matched on the basis of relevant socio-demographical variables (Table 1).

Table 1. Demographic characteristics and the equivalence of the groups

\begin{tabular}{lccccccc}
\hline \multirow{2}{*}{ Demography } & \multicolumn{2}{c}{ Controls $(\mathrm{N}=20)$} & \multicolumn{2}{c}{$\mathrm{SCH}(\mathrm{N}=26)$} & \multicolumn{2}{c}{ BAP $(\mathrm{N}=18)$} & \multirow{2}{*}{ Group difference } \\
& Mean & $\mathrm{SD}$ & Mean & $\mathrm{SD}$ & Mean & $\mathrm{SD}$ & \\
\hline Age (years) & 34.1 & \pm 4.4 & 34.8 & \pm 6.8 & 38.8 & \pm 8.3 & $\mathrm{P}>0.05$ \\
Education (years) & 12.5 & \pm 1.9 & 12.3 & \pm 2.3 & 12.1 & \pm 2.9 & $\mathrm{P}>0.05$ \\
Hospitalizations & & & 3.1 & \pm 2.8 & 4.7 & \pm 5.0 & $\mathrm{P}>0.05$ \\
Sex (frequencies) & 7 & & & & & & \\
$\quad$ Male & 13 & & 7 & & 1 & & $\mathrm{P}>0.05$ \\
$\quad$ Female & & & & & 19 & & \\
\hline
\end{tabular}


Two clinical groups were also matched regarding the number of hospitalizations. The group equivalence was tested by the means of ANOVA and t-test for continuous variables and $\mathrm{CHI}$ square in the case of nominal variables.

\section{Methods}

The assessments were performed individually by experienced clinical psychologists. The following tests were applied:

\section{Wisconsin Card Sorting Test (WCST)}

The test requires of each subject to match cards from the decks to one of the four stimulus cards. The subject is given feedback each time whether he or she is right or wrong. Wisconsin Card Sorting Test (WCST) was developed to assess the capacity to form abstract concepts and to shift cognitive strategies in response to changing environmental conditions. (Berg 1948, Grant \& Berg 1948). Test requires several abilities, such as strategic planning, goal-oriented behavior and the ability to use environmental feedback to shift cognitive set (Heaton et al. 1993). The successful performance on WCST involves the maintenance of information about previous card sorts and their outcomes. Consequently, such a process places high demands on WM. Therefore, WCST performance is largely dependent on WM capacity (Hartman et al. 2001, 2003) and can be considered as a good indicator of WM. We used five separate measures of performance on WCST: total number of correct answers, total number of completed categories, number of first category trials, perseverative errors (the number of items in which the person persists in responding incorrectly despite the feedback) and conceptual level responses (consecutive correct responses occurring in runs of three).

\section{STROOP test}

In this test originally developed by Stroop (1935), participants are shown color words and are required to name the word or the color of the ink in which each word is printed. The word and the ink color can be congruent or incongruent. The test is a measure of cognitive control that assesses the ease with which a person can maintain a goal and suppress a dominant response in favor of a less common one. The Stroop task requires the ability to inhibit task-irrelevant information. The successful performance relies on WM capacity (Conway \& Engle 1994) enabling maintaining activation of relevant features and inhibition of those irrelevant. We used two indicators of Stroop task performance, number of correctly named words and number of correctly named colors.

\section{Digit Span forwards and backwards tasks}

A digit-span task (Wechsler 1944) was used to measure working memory's number storage capacity. Since the formulation of Baddeley and Hitch's theory, digit span task has been considered as a part of the phonological loop within the WM (Baddeley 1986, 2018). Subjects were asked to repeat strings of digits of increasing length (from 3 to 9) read aloud by the examiner in the same (forward) and in reverse (backward) order. The measure of performance was the longest string of correctly recalled digits.

\section{Trail Making Test}

Trail Making Test (TMT) (Reitan 1958) is usually defined as a measure of attention, speed, mental flexibility and WM. The test requires the subject to connect, by making pencil lines, 25 encircled numbers randomly arranged on a page in proper order (Part A) and 25 encircled numbers and letters in alternating order (Part B). The performance on TMT involves activation of WM, especially in part B when subject must maintain in memory both numbers and letters. The measure of performance was time needed to complete task $\mathrm{A}$ and $\mathrm{B}$ in seconds.

\section{Statistical analysis}

All statistical analyses were performed using SPSS/PASW and AMOS version 20 (IBM Corp. N.Y. USA). To test normality of distributions, we calculated both Kolmogorov-Smirnov and Shapiro-Wilk tests. Unlike majority of distributions that did not significantly depart from normality, those related to WCST were mostly skewed. Therefore, to determine if group means belong to the same population we calculated both ANOVA and Kruskal-Wallis tests. The differences were considered statistically significant at $p$ values lower than 0.05. In addition, we used EFA and CFA to determine if used tests are indicators of the same cognitive ability (WM) or are saturated with different abilities. To identify the underlying dimensionality of used tasks, we used parallel analysis (Horn 1965).

\section{RESULTS}

A summary of means, standard deviations and group differences is given in table 2 .

Analyses show that ANOVA and Kruskal-Wallis test point to similar conclusions regardless of the distribution normality. Most indicators of the WCTS do not differentiate between healthy controls and the two clinical groups. Only the number of perseverative errors is found to be statistically different between controls and SCZ group. Other tests that were applied in the current study can distinguish controls from one or both clinical groups. On STROOP tasks as well as on TMT A and digit span forward, both SCZ and BPD group score significantly lower than controls. TMT B and digit span backward can differentiate only BPD from controls. The difference between SCZ and BPD group were never found to be statistically significant. Group profiles of scores transformed into z-values are given in Figure 1. Better performance is always displayed with positive values. 
Table 2. Descriptive statistics and comparison of test means between groups

\begin{tabular}{|c|c|c|c|c|c|c|c|c|c|c|}
\hline & \multicolumn{2}{|c|}{ Controls $(\mathrm{N}=20)$} & \multicolumn{2}{|c|}{$\mathrm{SCZ}(\mathrm{N}=26)$} & \multicolumn{2}{|c|}{$\operatorname{BAP}(\mathrm{N}=17)$} & \multicolumn{4}{|c|}{ Difference between groups } \\
\hline & M & SD & M & SD & M & SD & $\mathrm{F}$ & $\mathrm{p}$ & $\begin{array}{l}\text { Post hoc } \\
(p<0.05)\end{array}$ & $\begin{array}{l}\text { Kruskal- } \\
\text { Wallis p }\end{array}$ \\
\hline WCST correct answers & 79.3 & 14.1 & 67.3 & 18.0 & 72.6 & 16.9 & 2.96 & 0.059 & & 0.140 \\
\hline WCST perseverative errors & 8.1 & 7.9 & 29.3 & 25.9 & 16.2 & 22.0 & 6.14 & 0.004 & $\mathrm{C}-\mathrm{SCZ}$ & 0.002 \\
\hline WCST conceptual level & 62.5 & 11.9 & 51.8 & 23.6 & 60.7 & 25.0 & 1.72 & 0.188 & & 0.101 \\
\hline WCST categories completed & 4.8 & 1.7 & 3.6 & 2.3 & 4.5 & 2.5 & 1.86 & 0.164 & & 0.152 \\
\hline WCST first category trials & 13.9 & 8.6 & 38.6 & 43.2 & 35.8 & 46.6 & 2.80 & 0.069 & & 0.034 \\
\hline STROOP words & 265.6 & 27.9 & 212.2 & 57.8 & 207.8 & 56.1 & 8.37 & 0.001 & $\begin{array}{l}\text { C-SCZ; } \\
\text { C-BAP }\end{array}$ & 0.001 \\
\hline STROOP colors & 112.8 & 21.2 & 80.5 & 27.2 & 79.7 & 22.0 & 12.43 & $<0.001$ & $\begin{array}{l}\text { C-SCZ; } \\
\text { C-BAP }\end{array}$ & $\mathrm{p}<0.001$ \\
\hline TMT A (seconds) & 35.7 & 10.4 & 67.0 & 41.8 & 73.8 & 51.8 & 5.52 & 0.006 & $\begin{array}{l}\text { C-SCZ; } \\
\text { C-BAP }\end{array}$ & 0.002 \\
\hline TMT B (seconds) & 70.9 & 14.6 & 138.2 & 102.5 & 200.8 & 175.7 & 6.15 & 0.004 & C-BAP & $\mathrm{p}<0.001$ \\
\hline WAIS digit span forward & 11.0 & 2.0 & 7.9 & 1.9 & 7.8 & 2.4 & 15.80 & $<0.001$ & $\begin{array}{l}\text { C-SCZ; } \\
\text { C-BAP }\end{array}$ & $\mathrm{p}<0.001$ \\
\hline WAIS digit span backwards & 7.2 & 2.4 & 5.9 & 1.8 & 5.2 & 2.2 & 4.63 & 0.013 & C-BAP & 0.032 \\
\hline
\end{tabular}

$\begin{array}{llllllll}\text { Notes: WCST = Wisconsin Card Sorting Test; } & \text { TMT = Trail Making Test; } \quad \text { WAIS = Wechsler Adult Intelligence Scale }\end{array}$
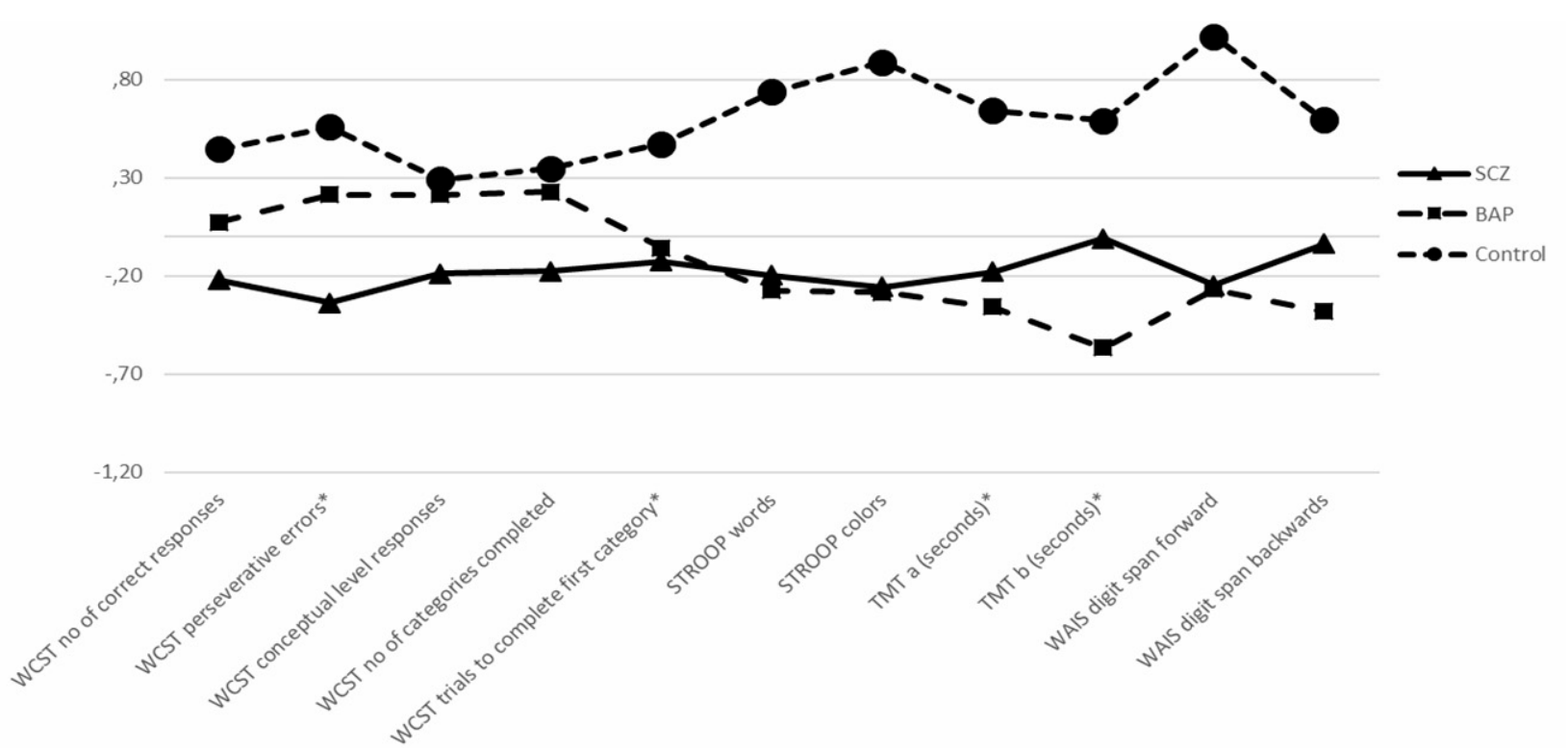

Notes: $\mathrm{SCZ}=$ schizophrenia $(\mathrm{N}=26) ; \quad \mathrm{BPD}=$ bipolar affective disorder $(\mathrm{N}=18) ; \quad$ Control = no psychiatric diagnosis $(\mathrm{N}=20)$;

* inverted results: larger scores indicate better performance; WCST = Wisconsin Card Sorting Test; TMT = Trail Making Test; WAIS = Wechsler Adult Intelligence Scale

Figure 1. Comparison of standardized means of WM indicators between groups

Since we found no statistically significant difference between SCZ and BPD group, results provide no evidence of difference in profile of working memory impairment. However, results do suggest that the impairment in some areas may be more serious in one group than in another when compared to healthy controls. Results also indicate that the difference between controls and clinical groups is smaller in the case of WCST than in other tests that were applied. This difference may reflect the difference in saturation with WM. To test if all indicators can be explained with a single broad factor, we conducted both EFA and CFA. Given a small number of respondents, the results can be considered only provisional. Prior to factorization, we ran Parallel analysis to deter- mine underlying dimensionality. The analysis pointed out that intercorrelations of the indicators can be explained with two underlying dimensions. Table 3 presents the standardized factor loadings of the WM indicators on these dimensions. The significant projections on the first component show only indicators belonging to WCST. All other indicators are highly saturated with the second component. Given that the main feature of the WCST is the ability to focus on the task, maintain previously gained information and override a tendency to repeatedly provide dominant but incorrect response, is tentatively named Working Memory Attention. The second component comprises of indicators commonly related to the size of a memory store and is therefore named Working Memory Capacity. 
Table 3. Varimax rotated components of the working memory indicators $(\mathrm{N}=64)$

\begin{tabular}{lcc}
\hline & WM Attention control & WM Capacity and speed \\
\hline WCST no of correct responses & 0.86 & \\
WCST perseverative errors & -0.75 & \\
WCST conceptual level responses & 0.95 & \\
WCST no of categories completed & 0.86 & \\
WCST trials to complete first category & -0.90 & 0.81 \\
STROOP words & & 0.71 \\
STROOP colors & & -0.84 \\
TMT a (seconds) & & -0.79 \\
TMT b (seconds) & & 0.74 \\
WAIS digit span forward & & 0.60 \\
\hline WAIS digit span backwards & & \\
\hline
\end{tabular}

Notes: Projections bellow 0.30 are omitted

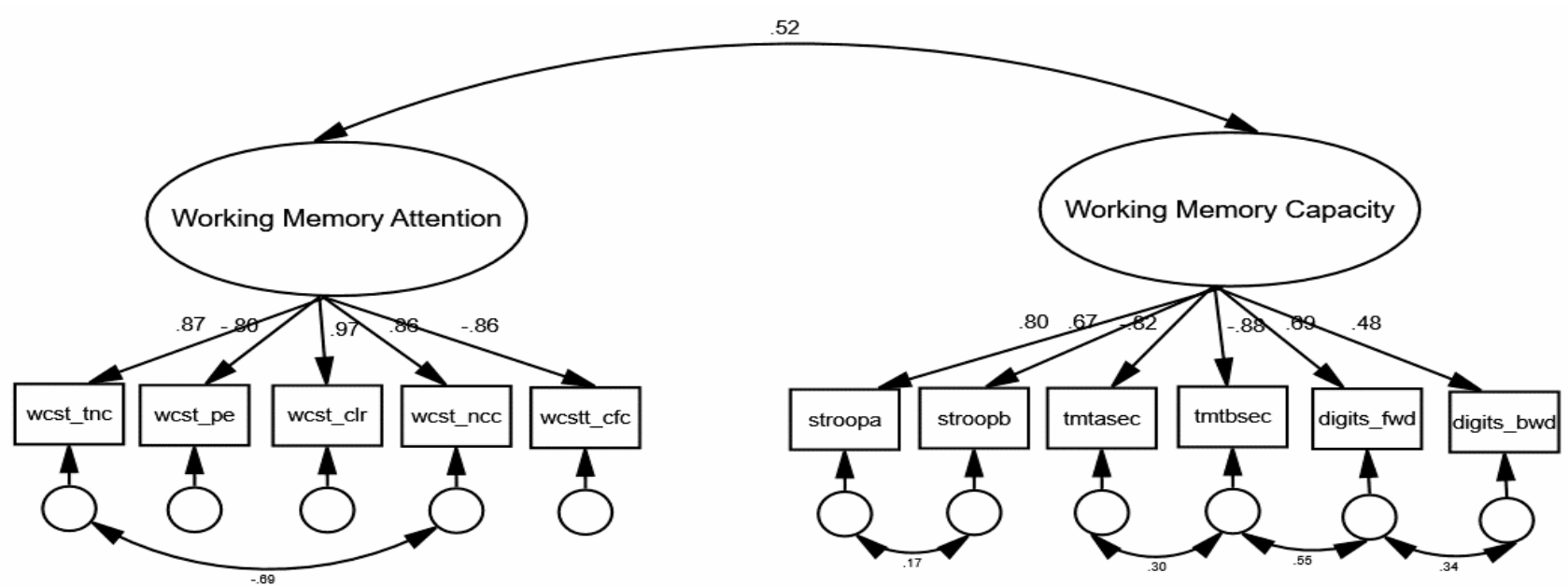

Fit indicators: $\chi^{2}(\mathrm{df} 38, \mathrm{~N}=66)=49.97, \mathrm{p}=0.093 ; \mathrm{CFI}=0.98, \mathrm{RMSEA}=0.072,90 \% \mathrm{CI}(0.000,0.12) ; \quad \mathrm{GFI}=0.88$; AGFI $=0.79 ; \quad$ NFI $=0.91$

Figure 2. Latent structure of WM indicators used in the current study

To provide a further test of a latent structure underlying WM indicators used in the current study, we applied CFA. The proposed two-factor model (Figure 2) yielded some acceptable indices of fit $\chi^{2}(\mathrm{df} 38, \mathrm{~N}=66)=49.97$, $\mathrm{p}=0.093, \mathrm{CFI}=0.98, \mathrm{RMSEA}=0.072,90 \%$ CI $(0.000$, 0.12 ) whereas others indicated a poor fit: $\mathrm{GFI}=0.88$, AGFI $=0.79, \mathrm{NFI}=0.91$.

Given that determining the structure of indicators was not the main topic of the current study, we concluded that applied tests are saturated with at least two moderately correlated latent dimensions, provisionally named WM Attention and WM Capacity. To avoid psychometric weaknesses of analyses on single indicators, we calculated factor scores and thus gained more reliable composites. An additional advantage was a greater parsimony since applied indicators were reduced to only two components. ANOVA performed on factor scores indicated that three groups did not differ on the Attention factor $(\mathrm{F}=1.31 ; \mathrm{p}>0.05)$. The statistically significant difference was found only on the Capacity factor $(\mathrm{F}=13.22$; $\mathrm{p}<0.001)$. Post hoc tests indicated that both clinical groups scored lower than controls, however, no significant difference was found between SCZ and BPD patients.

\section{DISCUSSION}

Previous research suggests that cognitive deficits may be considered as one of the most salient features of both SCZ (Barch \& Sheffield 2014) and BPD (MacQueen \& Memedovich 2017). Moreover, it is hypothesized that WM may underlie most of cognitive deficits found in psychosis and is probably responsible for the most prominent features of the disorder. Whereas the researchers unanimously agree that WM of people suffering from psychosis is impaired compared to healthy individuals, there is an ongoing dispute among authors regarding the potential differences in WM between those suffering from SCZ and those with BPD. Most of the evidence suggest that the WM of persons with $\mathrm{SCZ}$ is impaired more than the WM of those diagnosed with BPD, however it is not clear whether this difference is just of quantitative or also of qualitative nature (Barch \& Sheffield 2014). Answering these questions could have broader repercussions on the notion of these two disorders being regarded as two entirely distinct entities.

Accordingly, current study was initiated to determine whether the profile of WM impairment is the same 
for persons suffering from SCZ and those with BPD. In accordance with the claims of some authors who believe that persons with SCZ and those with BPD share the same profile of WM impairment (Barch \& Sheffield 2014), we found no difference between the two in performance on any of applied tests. There are, however, three indicators that differentiated healthy controls from one clinical group, but not from the other. Perseverative errors within WCST were more frequent among subjects with SCZ compared to control group, whereas participants with BPD did not differ from either group. By contrast, BPD patients performed poorly on TMT B task and Digit span backward in comparison to healthy controls whereas SCZ group could not be statistically distinguished from either group. These differences may be a reflection of some profile differences between persons with SCZ diagnosis versus those with BPD, but it is also possible that they are obtained by chance due to the limited sample size. Current research, unlike some previous studies (Krabbendam et al. 2005, Hill et al. 2013) thus provided no evidence of SCZ patients having more impaired WM than those suffering from BPD. The research has also provided no evidence that profiles of WM impairment in patients with SCZ and those with BPD differ. This finding is in line with the belief held by some authors that the WM impairment in SCZ and BPD patients compared to healthy population, follows the same profile (Tamminga et al. 2014, Barch \& Sheffield 2014, Šoštarič \& Zalar 2011). The current study has undeniably proved only that both clinical groups show impairment of WM compared to healthy controls. In addition to analysis of individual test results, we have also performed factor analysis to reduce the complexity of measures and gain parsimony. The results suggest that applied tests are saturated with two components, provisionally named WM attention and WM capacity. Only the latter produced significant difference between groups, indicating that both patients with SCZ and those with BPD show impairments in comparison to controls, but no difference was found between the two clinical groups.

The obtained results are thus in line with the assumption that there is no difference in the profile of WM impairment between persons with SCZ compared to those with BPD. Although these findings seemingly contradict the notion of SCZ and BPD being distinct entities, they only indicate that WM has limited or no value in discriminating between these two categories of psychotic disorders. Accordingly, the obtained results support the assumption that these two entities may share common genes responsible for cognitive impairments (Craddock et al. 2006, Maier et al. 1993). However, further research is needed, especially longitudinal tracking in order to determine the growth curve of WM deterioration in SCZ compared to BPD as some previous research found difference in their latent trajectories (Lewandovski et al. 2011). The current study is subject to some limitations that deserve attention. First, study has been carried out on rather small sample, therefore, results are lacking in statistical power. The findings would have been much clearer if larger groups were included. Second, cross-sectional design applied in the study cannot provide information on the timeframe of change and the dynamics of deterioration of WM in SCZ and BPD. Therefore, in the future it would certainly be worthwhile to carry out longitudinal research, Third, given that WM is a complex construct that affects almost all cognitive processes, it is not easy to capture it. Probably there is no test that reflects exclusively the working memory, as there is no cognitive test that does not include the WM component. Arguably, many of the tests that we used only partly reflect WM while the rest is saturated with other cognitive abilities. The observed differences between the groups may reflect differences in WM but may also reflect differences in other cognitive abilities. That is why construct validity might have been compromised. Further research on a larger sample of respondents and with larger number of tests is needed to provide clearer evidence of WM in patients with SCZ and BPD.

\section{CONCLUSIONS}

The current study determined impairment of WM in both SCZ and BPD compared to control group. However, no difference was found regarding either the magnitude or the profile of impairment between SCZ and BPD patients. Further research on larger samples and with larger number of WM measures is needed to provide stronger evidence on the issue.

\section{Acknowledgements:}

We sincerely thank all study participants for taking part in this study as well as to clinical psychologists for helping in testing and data collection.

\section{Conflict of interest: None to declare.}

\section{Contribution of individual authors:}

Danijela Žakić Milas conceptualized and designed the study, carried out data collection, participated in data interpretation and manuscript preparation;

Goran Milas performed statistical analyses and participated in data interpretation and manuscript preparation.

\section{References}

1. Baddeley AD \& Hitch GJ: Working memory. In Bower, GA (ed): The Psychology of Learning and Motivation: Advances in Research and Theory, 47-89. Academic Press, 1974

2. Baddeley AD: Working Memory. Oxford University Press, Oxford, 1986

3. Baddeley AD: Exploring Working Memory. Routledge, London, 2018 
4. Barch DA: Cognition in schizophrenia: Does working memory work? Curr Dir Psychol Sci 2003; 12:146-50

5. Barch DM, Csernansky JG, Conturo T, Snyder AZ \& Ollinger J: Working and long-term memory deficits in schizophrenia. Is there a common underlying prefrontal mechanism? J Abnorm Psychol 2002; 111:478-94

6. Barch DM \& Sheffield JM: Cognitive impairments in psychotic disorders: common mechanisms and measurement. World Psychiatry 2014; 13:224-32

7. Barch DM, Sheline YI, Csernansky JG \& Snyder AZ: Working memory and prefrontal cortex dysfunction: Specificity to schizophrenia compared with major depression. Biol Psychiatry 2003; 53:376-84

8. Berg EA: Simple Objective Technique for Measuring Flexibilty in Thinking. J Gen Psychol 1948; 39:15-22

9. Braver TS, Barch DM \& Cohen JD: Cognition and control in schizophrenia: A computational model of dopamine and prefrontal function. Biol Psychiatry 1999; 46:312-28

10. Braver TS, Paxton JL, Locke HS \& Barch DM: Flexible neural mechanisms of cognitive control within human prefrontal cortex. Proc Natl Acad Sci USA 2009; 106:7351-56

11. Cohen JD, Barch DM, Carter CS \& Servan-Schreiber D: Schizophrenic deficits in the processing of context: Converging evidence from three theoretically motivated cognitive tasks. J Abnorm Psychol 1999; 108:120-33

12. Conway ARA \& Engle RW: Working memory and retrieval: A resource- dependent inhibition model. J Exp Psychol Gen 1994; 123:354-73

13. Craddock N, O'Donovan MC \& Owen MJ: Genes for schizophrenia and bipolar disorder? Implications for psychiatric nosology. Schizophr Bull 2006; 32:9-16

14. Edwards BG, Barch DM \& Braver TS: Improving prefrontal cortex function in schizophrenia through focused training of cognitive control. Front Hum Neurosci 2010; 4:32

15. Engle RW: Working Memory Capacity as Executive Attention. Psychol Sci 2002; 11:19-23

16. Forbes NF, Carrick LA, McIntosh AM \& Lawrie SM: Working memory in schizophrenia: A meta-analysis. Psychol Med 2009; 39:889-905

17. Glahn DC, Bearden CE, Cakir S, Barett JA, Najt P, Monkukl ES et al: Differential working memory impairment in bipolar disorder and schizophrenia: Effects of lifetime history of psychosis. Bipolar Disord 2006; 8:117-23

18. Gold JM, Robinson B \& Leonard CJ: Selective Attention, Working Memory, and Executive Function as Potential Independent Sources of Cognitive Dysfunction in Schizophrenia. Schizophr Bull 2017; 1:1-8

19. Goldman-Rakic PS: Working memory dysfunction in schizophrenia. J Neuropsychiatry Clin Neurosci 1994; 6:348-57

20. Goldman-Rakic PS: Prefrontal cortical dysfunction in schizophrenia: The relevance of working memory In Carrol BJ \& Barret JE (eds). Psychopathology and the brain, 1-23 Raven Press, 1991

21. Grant DA \& Berg E: A behavioral analysis of degree of reinforcement and ease of shifting to new responses in a Weigl-type card-sorting problem. J Exp Psychol 1948; 38:404-11

22. Green MF, Kern RS, Braff DL \& Mintz J: Neurocognitive Deficits and Functional Outcome in Schizophrenia:
Are We Measuring the "Right Stuff"? Schizophr Bull 2000; 26:119-36

23. Hartman M, Bolton E \& Fehnel SE: Accounting for age differences on the Wisconsin Card Sorting Test: decreasing working memory, not inflexibility. Psychol Aging 2001; 16:299-385

24. Hartman M, Steketee MC, Silva S, Lanning $K$ \& Andersson C: Wisconsin Card Sorting Test performance in schizophrenia: The role of working memory. Schizophr Res 2003; 63:201-17

25. Heaton RK, Chelune GJ, Talley JL, Kay GG \& Curtis G: Wisconsin Card Sorting Test (WCST) manual, revised and expanded. Psychological Assessment Resources, Odessa, 1993

26. Hill SK, Reilly JL, Keefe RSE, Gold JM, Bishop JR, Gershon ES et al: Neuropsychological Impairments in Schizophrenia and Psychotic Bipolar Disorder: Findings from the Bipolar and Schizophrenia Network on Intermediate Phenotypes (B-SNIP) Study. Am $J$ Psychiatry 2013; 11:1-10

27. Horn JL: A rationale and test for the number of factors in factor analysis. Psychometrika 1965; 30:179-85

28. Krabbendam L, Arts B, Van Os $J \&$ Aleman A; Cognitive functioning in patients with schizophrenia and bipolar disorder: A quantitative review. Schizophr Res 2005; 80:137-49

29. Lee $J$ \& Park S: Working memory impairments in schizophrenia: a meta-analysis. J Abnorm Psychol 2005; 114:599-611

30. Lesh TA, Niendam TA, Minzenberg MJ \& Carter, CS: Cognitive control deficits in schizophrenia: mechanisms and meaning. Neuropsychopharmacology 2011; 36:316-38

31. Lewandowski KE, Cohen BM \& Ongur D: Evolution of neuropsychological dysfunction during the course of schizophrenia and bipolar disorder. Psychol Med 2011; 41:225-41

32. MacQueen GM \& Memedovich KA: Cognitive dysfunction in major depression and bipolar disorder: Assessment and treatment options. Psychiatry Clin Neurosci 2017; 71:18-27

33. Maier W, Lichtermann D, Minges J, Hallmayer J, Heun $R$, Benkert $O$ et al: Continuity and discontinuity of affective disorders and schizophrenia: Results of a controlled family study. Arch Gen Psychiatry 1993; 50:871-83

34. Park $S \&$ Gooding DC. Working memory impairment as an endophenotypic marker of a schizophrenia diathesis. Schizophr Res Cogn 2014; 1:127-36

35. Park S, Holzman PS \& Goldman-Rakic PS: Spatial working memory deficit in relatives of patients with schizophrenia. Arch Gen Psychiatry 1995; 52:821-28

36. Park $S$ \& Holzman PS: Schizophrenics show spatial working memory deficits. Arch Gen Psychiatry 1992; 49:975-82

37. Pflueger MO, Calabrese P, Studerus E, Gschwandtner $U$, Borqwardt S, Aston $J$ et al: The neuropsychology of emerging psychosis and the role of working memory in episodic memory encoding. Psychol Res Behav Manag 2018; 11:157-68

38. Reitan RM: Validity of the Trail Making Test as an Indicator of Organic Brain Damage. Percept Mot Skills 1958; 8:271-76 
39. Stroop JR: Studies of Interference in Serial Verbal Reactions. J Exp Psychol 1935; 18:643-62

40. Šoštarič $M \&$ Zalar B: The overlap of cognitive impairment in depression and schizophrenia: A comparative study. Psychiatr Danub 2011; 23:251-56

41. Tamminga C, Pearlson G, Keshavan M, Sweeney J, Clementz B \& Thaker G: Bipolar and schizophrenia net- work for intermediate phenotypes: Outcomes across the psychosis continuum. Schizophr Bull 2014; 40:131-37

42. Wechsler D: The measurement of adult intelligence (3rd ed). Williams \& Wilkins, Baltimore, 1944

43. World Health Organization: The ICD-10 classification of mental and behavioural disorders: Clinical descriptions and diagnostic guidelines. WHO, Geneva, 1993

\section{Correspondence:}

Professor Goran Milas, PhD

Institute of Social Sciences "Ivo Pilar"

Marulićev trg 19, 10000 Zagreb, Croatia

E-mail:goran.milas@pilar.hr 